\title{
Psychophysiological features of solving mathematical examples with fractions as adaptability markers to the cognitive load
}

\author{
Anna Fomina ${ }^{1 *}$ and Kristina Ganusha ${ }^{2}$ \\ ${ }^{1}$ Don State Technical University, 344000, Rostov-on-Don, Russia \\ ${ }^{2}$ Southern Federal University, 344090, Rostov-on-Don, Russia
}

\begin{abstract}
This study is devoted to identifying a solution algorithm for standard fractions as one of the tasks that allow investigating the level of human adaptability to the cognitive load. The influential factor for a successful solution was the number of stages, and for an unsuccessful one their duration. It was revealed that the solution success/failure correlated with the spectral power values and ratio in the theta- and alpha-diapasons of the EEG. The successful solution is accompanied by the maintenance of a stable level of theta-diapason and desynchronization in the alpha-diapason. The unsuccessful solution is characterized by an increase in the thetadiapason power, its shift to the frontal zones, and a lack of alphadesynchronization.
\end{abstract}

\section{Introduction}

The assessment of the personal adaptability level to performing complex compound intellectual activity assumes individual importance in the context of the transition to distance learning with the use of digital technologies. Adaptability is estimated as the ability to choose the correct strategy for a task solution, regarding its structure and requirements for quality specifications [1-4]. Arithmetic problems solving is considered as a promising way of evaluating the adaptability level since the formed skill of calculations significantly correlates with the acquisition progress for natural science disciplines in secondary and higher education [3-7]. The high adaptability level to performing complex calculations makes it possible to assess the ability of a person to select and apply optimal strategies for activities in other areas [2].

Fractional calculations are of particular importance since mathematical problems are used to assess the adaptability level. This task type is a transitional stage from simple arithmetic operations to complex algebraic calculations and statistical analysis [5] and requires the use of a rigid algorithm of operating procedures $[6,8]$. The tasks solving errors with fractions might be connected with both deviations from this algorithm and with cognitive abilities, namely volitional attention, working memory [9], as well as mental arithmetic skills.

The main challenge in the application of mathematical problems is the absence of external correlates of the solution process, which might become the basis for possible algorithms for

\footnotetext{
*Corresponding author: a_bogun@mail.ru
} 
performing the task and identifying possible reasons for errors. Using the dual-task paradigm, we managed to obtain algorithms for solving examples for the addition and multiplication of two-digit numbers taking into account the number and duration of intermediate stages [10]. Such studies for fractional numbers had not been carried out before due to the significant complexity of the activity.

This study is devoted to the analysis of psychophysiological features during the solution mathematical examples of standard fractions addition and division to assess an adaptability level to the cognitive load.

\section{Materials and methods}

The study involved 30 students of the Southern Federal University, of 20 - 22 years old (23 women, 7 men, average age 20,9 $\pm 2,9)$. The experiments were carried out in a dark soundproof room, in which the individuals tested seated in front of the computer. All subjects were informed about the order of the testing procedures and signed a written agreement for the tests to be performed in accordance with ethical standards.

At the beginning of the study, EEG was recorded in a state of quiet wakefulness with open eyes for 5 minutes. Then the participants were given instructions on how to perform the required task and familiarized with the interface and its control keys. The major research technique consisted of 2 parts - 'Fractions Addition' and 'Fractions Division'. Every block contained 30 examples; the arithmetic operation sign inside the block was not changed. Slides with examples printed in black (Times New Roman, 80 type size) were used as incentives. The examples were presented sequentially at regular intervals. The period of time for the arithmetic operations solution was selected individually for every person. All task keys were typed in a standard type MS Word file.

Students were provided with the following instruction: press the mouse-manipulator button after reading the task, before starting to type the answer, and after finishing the task (Fig. 1).

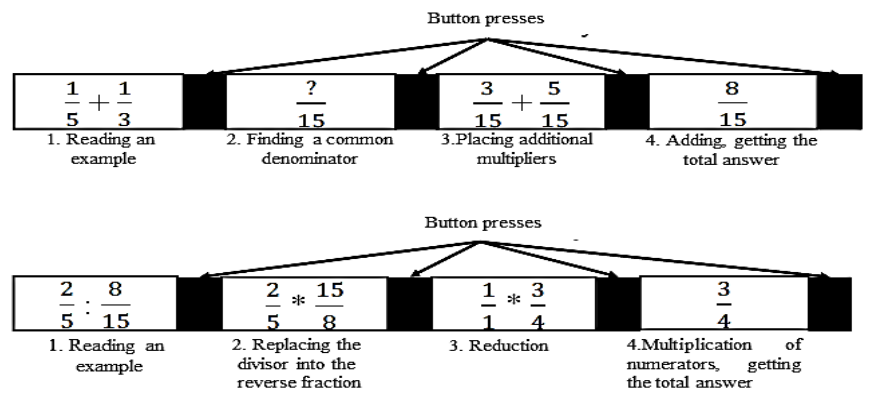

Fig. 1. Stages of a fraction task solving

Stimuli were represented using the "Audiovisual slider" program (Medikom-MTD, Russia) at the grey background in the center of the computer screen at $1 \mathrm{~m}$ distance at the eye level. Electrophysiological parameters were registered with the multichannel computer-based encephalograph "Encephalan-131-03" (Medikom-MTD, Russia). Reference electrodes were placed upon earlaps; the indifferent electrode was located on the forehead. Digitalized EEG, as well as the stimuli and button press markers, were exported into the MATLAB for further processing. The time required to solve individual tasks of each type, duration of each stage, the number of terms used, and the values of spectral power of four EEG diapasons were analyzed: delta $(1-3.5 \mathrm{~Hz})$, theta $(4-8 \mathrm{~Hz})$ alpha $(8-13 \mathrm{~Hz})$ and beta $(13-30 \mathrm{~Hz})$.

Spectral power was analyzed by the artifact-free EEG-segments. Confidence of differences was assessed by ANOVA at the significance level of 0.05 . Holm and Tukey 
methods were used to correct multiple comparisons and the Greenhouse-Geisser correction was used to correct the number of freedom degrees. The statistical analysis of the EEG spectral power (SP) was performed for the zones selected: frontal, central, temporal, parietal, and occipital. The dispersion analysis was carried out considering the following factors: CONTENT (addition or division task), CORRECTION (correct or incorrect answer), and LOCATION (five zones selected). The factor of the CONDITION, which included the comparative analysis of the text with the background, was considered individually. Individual differences were considered as random factors.

\section{Results}

To analyze the differences between correct and incorrect examples solutions, it was decided to divide all examples into blocks related to the correctness of the solution. Statistical analysis demonstrated a valid effect on the time solution values of the factors CONTENT $(\mathrm{F}(1,1)=15,431, \mathrm{p}=0,00009)$ и СORRECTION $(\mathrm{F}(1,1)=5,1617, \mathrm{p}=0,02327)$.

The analyzes demonstrates that the time taken for solving examples of fractions addition correctly is evidently less than the time taken for the incorrect ones $(37,6 \pm 0,8 \mathrm{~s}$. and $44 \pm 1,3$ s.). The part of correctly solved examples was $71.6 \%$, and incorrectly ones $-28.4 \%$. As for division examples, the time period for correct and incorrect solutions did not differ significantly $(31.7 \pm 0.7 \mathrm{~s}$ and $32.6 \pm 1.4 \mathrm{~s}$, accordingly). The part of correctly solved examples was $72.6 \%$, and incorrectly ones $-27.4 \%$. Compared to the examples of fractions addition, the solution time for solving examples of fractions division was statistically less without reference to correctness.

It should be noted that the analysis of the time period taken for solving examples immediately after the wrong answer did not show significant fluctuations in the values of this indicator compared to the average correct or incorrect solution ().

The average time for solving examples of fractions addition and division is shown in Table 1 during the solution in a different number of stages. The CORRECTION factor (F $(1,1)=48.836, p=0.00001)$ influenced reliably on the number of stages with a separate action and the CONTENT * CORRECTION factors with the joint one $(\mathrm{F}(1,1,1)=10,142, \mathrm{p}$ $=0,00149)$. As we can see in the Figure, the time for solving examples of fractions addition with the correct answer was significantly shorter compared with the wrong answer when two, three, and four stages were selected. The time for solving examples in the single stage did not differ. The correct answer was accompanied by an increasing number of stages. The main part of the examples was solved correctly in two, three, and four stages but mistakenly in one, two, and three stages.

As for fractions division, significant differences in the time solution were registered only during the three stages of solving $(\mathrm{F}(1,1)=24.548, \mathrm{p}=0.00001)$; there were not found any differences for the rest of the combinations. The main part of the examples was solved correctly in two, three, and four stages but mistakenly in two and three stages.

Table 1. Time period for solving examples of fractions addition and division depending on the correct answer and number of stages

\begin{tabular}{|c|c|c|c|c|}
\hline \multirow{2}{*}{$\begin{array}{c}\text { Number of } \\
\text { stages }\end{array}$} & \multicolumn{4}{|c|}{ Solution time, $\mathrm{s}$} \\
\cline { 2 - 5 } & Correct & Incorrect & Correct & Division \\
\cline { 2 - 5 } & $36.5 \pm 3.9$ & $33.5 \pm 3.5$ & $27.5 \pm 2.3$ & $30.2 \pm 4.6$ \\
\hline 1 & $39.7 \pm 1.8$ & $46 \pm 2.2^{*}$ & $29.1 \pm 1.4$ & $28.5 \pm 2.4$ \\
\hline 2 & $38.1 \pm 1.5$ & $47.5 \pm 2.2^{*}$ & $32.5 \pm 1.1$ & $36.9 \pm 2.3^{* *}$ \\
\hline 3 & $35.2 \pm 1.7$ & $45.2 \pm 3^{*}$ & $32.8 \pm 1.5$ & $35 \pm 2.4$ \\
\hline 4 & \multicolumn{3}{|c}{}
\end{tabular}


* differences in the solution time of addition examples are reliable at a significance level of $\mathrm{p} \leq 0.01$

** differences in the solution time of division examples are reliable at a significance level of $\mathrm{p} \leq 0.05$

Further analysis was treated for groups of examples solved in a different number of stages. The duration of single stages solving examples for adding and dividing fractions is represented in Figure 4. At the first stage the values of duration were statistically influenced by the CONTENT $(1, \mathrm{~F}(1,1)=54.5, \mathrm{p}=0.0015)$ and CORRECTION factors $(1, \mathrm{~F}(1,1)=$ $27.2, \mathrm{p}=0.0001)$. The duration of the second stage was statistically influenced by the CONTENT $((1, \mathrm{~F}(1,1)=28.469, \mathrm{p}=0.00001)$ and CORRECTION factors $(1, \mathrm{~F}(1,1)=$ $15.971, \mathrm{p}=0.00007)$ for separate and combined operations $(1, \mathrm{~F}(1,1,1)=9.6814, \mathrm{p}=$ $0.00191)$.

The linear dynamic of the duration stages was observed in solution examples at stages 2 and 3 without reference to the task and the correct answer. The highest values of duration are registered at the final stages. The dome-shaped dynamic was revealed during solution division examples in 4 stages and incorrectly solved addition examples.

The examples were calculated correctly in 3 stages, irrespectively from the task, and incorrectly - in 3 stages. The complete implementation of the computation algorithm corresponded to the execution of 4 stages.

Table 2. The duration of single stages solving examples for adding and dividing fractions

\begin{tabular}{|c|c|c|c|c|c|}
\hline \multirow{2}{*}{$\begin{array}{c}\text { Number of } \\
\text { stages }\end{array}$} & \multirow{2}{*}{ \# stage } & \multicolumn{4}{|c|}{ Duration of stages, s } \\
\cline { 3 - 6 } & & \multicolumn{2}{|c|}{ Addition } & \multicolumn{2}{c|}{ Division } \\
\cline { 3 - 6 } & Correct & Incorrect & Correct & Incorrect \\
\hline 2 & 1 & $12 \pm 1.5$ & $10.2 \pm 1.8$ & $8.1 \pm 1$ & $6.5 \pm 0.9$ \\
\cline { 2 - 6 } & 2 & $27.7 \pm 1.8$ & $35.8 \pm 2.5$ & $21 \pm 1.4$ & $22 \pm 2.2$ \\
\hline 3 & 1 & $7 \pm 0.6$ & $5.7 \pm 0.8$ & $4 \pm 0.4$ & $4.9 \pm 0.9$ \\
\cline { 2 - 6 } & 2 & $16 \pm 1$ & $20.7 \pm 2.2$ & $10.8 \pm 0.8$ & $8.3 \pm 1$ \\
\cline { 2 - 6 } & 3 & $15.2 \pm 0.9$ & $21.1 \pm 2.1$ & $17.7 \pm 1.1$ & $23.7 \pm 2.1$ \\
\hline 4 & 1 & $4 \pm 0.6$ & $7.8 \pm 1.9$ & $3.5 \pm 0.3$ & $5.2 \pm 1.7$ \\
\cline { 2 - 6 } & 2 & $10.8 \pm 1$ & $17.8 \pm 2.6$ & $5.8 \pm 0.8$ & $6.7 \pm 1.3$ \\
\cline { 2 - 6 } & 3 & $9.8 \pm 0.8$ & $11.2 \pm 1.7$ & $15 \pm 1$ & $13.1 \pm 1.5$ \\
\cline { 2 - 6 } & 4 & $10.6 \pm 0.9$ & $8.4 \pm 1$ & $8.5 \pm 0.8$ & $10 \pm 1$ \\
\hline
\end{tabular}



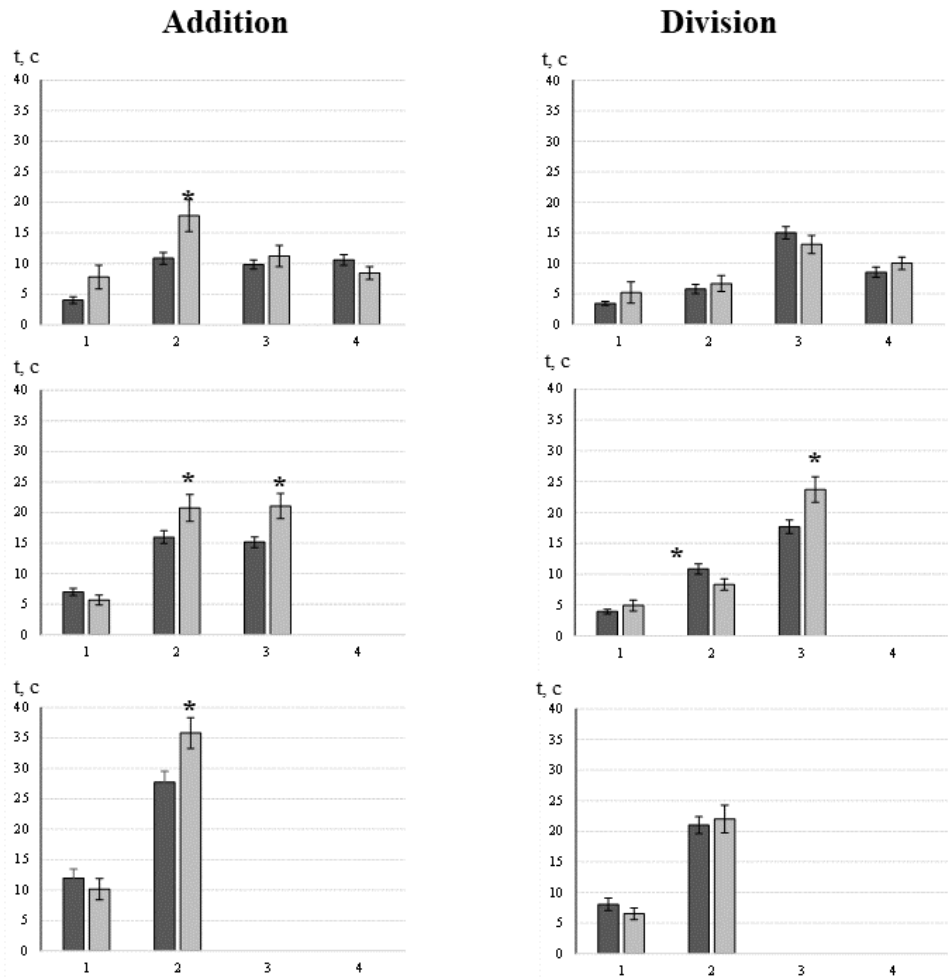

Designations: the number of solution stages is represented on the abscissa axis, the time solution period - on the axis of ordinates, $\mathrm{s}$.

Fig. 2. The duration of single stages solving examples for adding and dividing fractions depending on the solution correctness

The factors CONTENT $(\mathrm{F}(1,1)=11.218, \mathrm{p}=0.000818), \operatorname{LOCATION}(\mathrm{F}(1.19)=27.067$, $\mathrm{p}=0.000001)$, as well as the joint CONTENT $*$ CORRECTION factors $((\mathrm{F}(1,1,1)=18.865$, $\mathrm{p}=0.000014)$ influenced reliably on the power spectrum value of delta-frequencies. The factors CORRECTION $(\mathrm{F}(1,1)=54.39, \mathrm{p}=0.00012)$, LOCATION $(\mathrm{F}(1.19)=36.207, \mathrm{p}=$ $0.001)$ influenced reliably on the power spectrum value of theta-frequencies.

The factors CONTENT $(\mathrm{F}(1,1)=53.2, \mathrm{p}=0.00028)$, CORRECTION $(\mathrm{F}(1,1)=18.87$, $\mathrm{p}=0.01)$ and LOCATION $(\mathrm{F}(20.19)=63.119, \mathrm{p}=0.000001)$ influenced reliably on the power spectrum value of Alpha frequencies for separate operations, and CONTENT * CORRECTION factors for combined operations $(\mathrm{F}(1,1,1)=18.2, \mathrm{p}=0.004)$.

The topographic map of SP EEG values during the solution examples of fractions addition and division is represented in Figure 3. The desynchronization in the alpha-range and synchronization in the theta-diapason were observed during the correct calculation regardless of the task. There was registered a focus shift to the frontal zones in the delta-diapason. The focus moved to the frontal zones in the theta-diapason. There was also observed an emerging of mild focus in the occipital zones during the calculation examples of fractions addition. The focus shifted to the occipital zones, as well as evident desynchronization, revealed in the alpha-diapason.

If the calculation of examples was wrong, desynchronization occurred in all examined diapasons. The focus spread to the frontal zones in the delta-diapason. The focus shifts to the frontal zones occurred along with the significant desynchronization in the theta-diapason. 
The focus moved to the occipital zones in the alpha-diapason. As for the beta-diapason, the focus became symmetrical, expressed in the occipital zones.
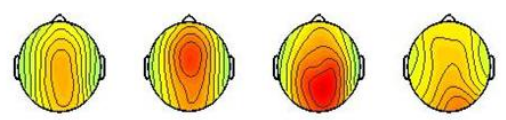

Open eyes

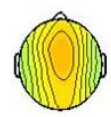

Addition
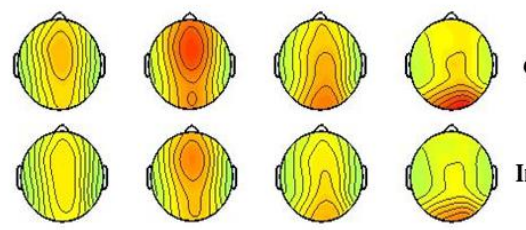

Correct
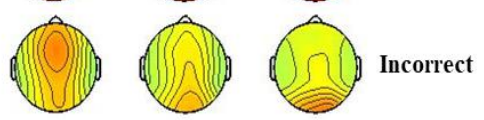

Division
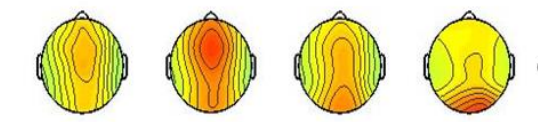

Correct
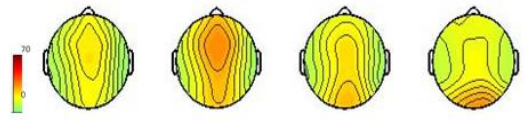

Incorrect

Delta

Theta

Alpha

Beta

Fig. 3. The topographic maps of the distribution of four spectral EEG diapasons in the state of quiet wakefulness with open eyes and during the task solving of all types. Note: functional probes are shown on the top; the corresponding rhythmic EEG diapason is shown on the down. The dark color indicates a high level of spectral power in the leads.

The distribution of the peak values of SP EEG during solution examples of fractions addition and division is exposed in Figure 4. As we can see in the figure, two frequency peaks were revealed in the theta-diapason spectrum, regardless of the task and correct calculation; one of the peaks was found in every tested area. The second peak was localized in the frontal zones (Fpz, Fz) when the solution was correct and in the frontal and central zones (Fpz, Fz, $\mathrm{Cz}$ ) with an incorrect solution. There was registered one frequency peak localized in the central parieto-occipital zones $(\mathrm{Cz}, \mathrm{Pz}, \mathrm{Oz}$, ) in the alpha-diapason spectrum.

The amplitude maximum of theta peak was observed when the addition examples were solved incorrectly. Therefore, regardless of the task and correct calculation, we might assume there are two theta-diapason generators and one alpha-diapason generator. 


\section{Addition}
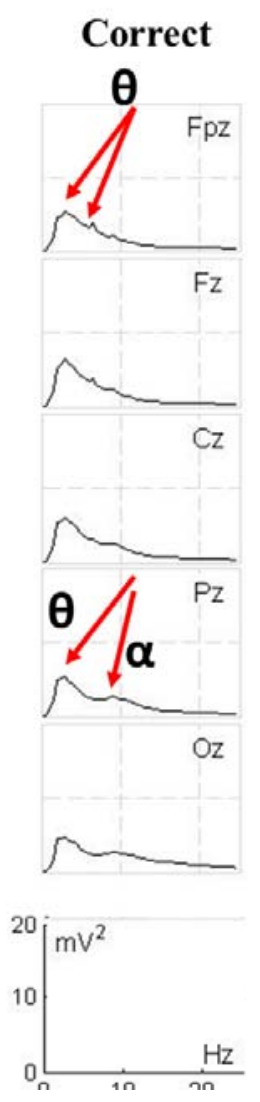

\section{Division}

\section{Incorrect}
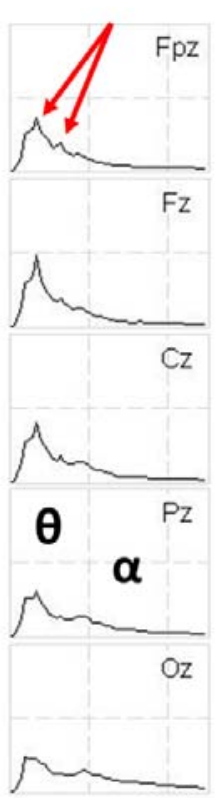

\section{Correct}

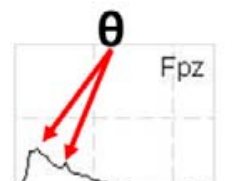

$\mathrm{Fz}$

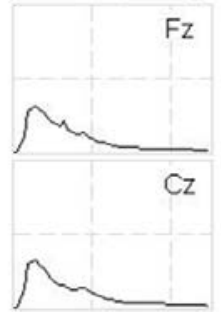

Incorrect
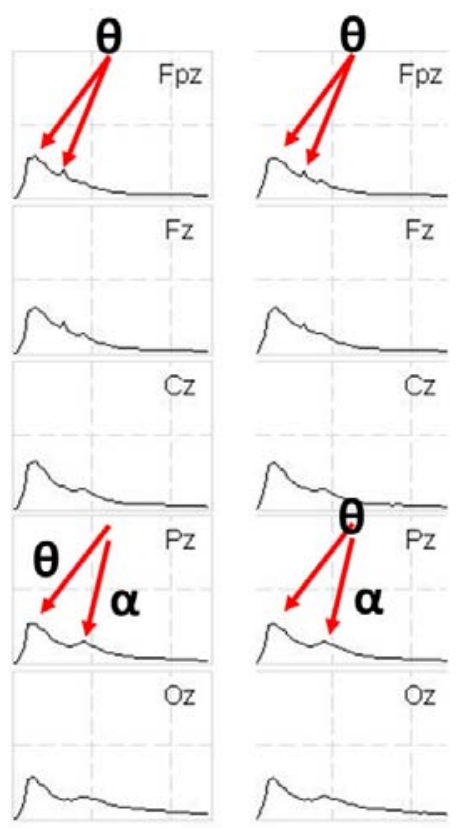

Fig. 4. Distribution of SP EEG peak values during solution examples of fractions addition and division. Note: Designations: the spectrum frequencies are represented on the abscissa axis; the spectral power values, $\mathrm{mV}$ - on the axis of ordinates

Thus, more activation is demonstrated with the correct solution for both tasks. The focus localization was similar regardless of the task and solution correctness. When the solution was correct a pronounced focus remained in the theta-diapason, regardless of the task. The weak focus was formed in the occipital zones while solving examples of fractions addition. The significant desynchronization and focus shift to the frontal zones appear with the wrong solution regardless of the task.

\section{Discussion}

Modern literature data confirm the possibility of using mathematical skills to assess the level of adaptability. The structure of the mathematical task itself and the possibility of adjusting the solution algorithm has a significant impact in addition to individual differences among survey participants. First of all, since the very ability to adjust is associated with the function of working memory and voluntary attention, it can be argued that these particular cognitive processes are crucial in assessing the adaptability level, and their impact on the dynamics of behavioral indicators will be the greatest.

Analyzing the solution stages, it was revealed that during addition operation, the greatest difficulty is caused by the execution of the second stage - the selection of a common factor 
[11]. Due to the specificity of the solution algorithm, the implementation of this stage is crucial, as its omission inevitably led to the incorrect answer. It can be assumed that the correct answer might require the optimal duration of the stages, and the reduction of the number of stages will affect only the last stages associated with the decision-making.

The implementation of the second and third stages, connected with replacing the divisor into the inverse fraction and reduction, did not cause any difficulties during solution examples of fractions division. Due to the fact that the solution process is started at the skimming stage of the example specification, just then a preliminary answer is getting received. The implementation of the subsequent stages is connected with the final computations.

The duration of the second stage for both tasks was significantly longer when the solution was incorrect, so it can be argued that its execution in the algorithm was critical. Difficulties at this stage led to getting the wrong answer.

Based on the obtained data about the solution time, the number, and duration of single stages, it can be assumed that there is an integration of stages for both tasks with partial overlap moving to a smaller number of them. As for the addition task, only the integration of the stages occurs, but during the division task, both the integration and the skipping of individual stages are possible. It can be assumed that the division problem is an easier task because it takes less time to solve than the addition one, and division contains a high percentage of correct answers [12].

At the same time, the dynamic analysis of the solution duration of single examples revealed the post-error slowing (PES) absence. PES means lengthening the time period for solving examples after identifying an error. Since PES is evidence of strengthening cognitive control [13-15], its absence implies not a change, but an adjustment of the dominant solution algorithm in the form of the changes in the number and/or duration of stages $[14,16]$. Consequently, the complexity and task content and, as a consequence, an increasing load of the working memory were reflected not in the quality, but in the solution speed $[17,18]$ and the number of stages.

There were not detected significant differences between the tasks in the SP EEG values analysis. It is connected with the formation of a certain activation level, which remained stable throughout the entire testing. Larger SP EEG values were shown with the correct solution, regardless of the task complexity. Based on the obtained data, it can be assumed that the correct solution requires a general level of common cortical activity and the main modulating systems [19,20 ]. The involvement of the fronto-parietal system and mental arithmetic is common in parallel with the activation of the prefrontal cortex associated with the working memory system [21], as well as reciprocal interactions of networks of calm and active wakefulness, acting as a predictor of the activation level of memory systems [21-24].

The contribution of various cognitive processes into the solution of both tasks was assessed on the SP EEG analysis results. The diffuse focus shaping in the delta-diapason and increasing SP EEG values are observed in the solution of all mathematical problems. It reflects the work of inhibiting mechanisms aimed at relevant information processing [25]. As the irradiation of delta frequencies occurred when both tasks were solved incorrectly, it can be regarded as a marker of challenge $[25,26]$. The increasing SP EEG values in the thetadiapason reflected the maintenance of volitional attention and changes in the load on working memory [20].

Maintaining a common level of theta-diapason with a correct solution, apart from the task, along with desynchronization in the alpha-diapason, might indicate the activation of the long-term memory system and a common level of the working memory load [27,28]. The reduction of the theta-diapason emerging can be associated with a decrease in cognitive control and difficulty focusing on the task [20,29-31]. With the solution is wrong more pronounced desynchronization of alpha-frequencies indicates a greater load on long-term memory. Probably, the solution of complex examples requires longer retention of numbers 
in the working memory, which makes the numerical processing harder [20,32,33]. As a result, it can be assumed that the adaptability to complex mental activity connected with the operating of the working memory system requires the active involvement of the prefrontal cortex, which is reflected in an increase in the SP EEG values for theta-frequencies.

There is a proposed scheme based on the obtained data (fig. 7). It reflects the adaptability level to task solving, as well as potential correlates of the process. The diagram shows that the simplest task, reflecting the minimum required level of adaptability, is the correct solution of examples for fractions division. The incorrect solution of division examples can be explained by the inefficient allocation of working memory resources, which does not allow to concentrate on the task and leads to the wrong answer. The correct solution of addition examples, as well as division ones, is associated with an even load on working and long-term memory. However, unlike division, the solution of addition examples is characterized by greater integration in theta-frequencies, which is associated with the higher complexity of the problem. The most difficult operation is the incorrect solution of addition examples and it is associated with the maximum complexity of the adaptation process.

Therefore, it can be argued that the effective solution of complex examples, in addition to the total high level of computation skills, is a marker of a high adaptability level, and errors in the solution of basic examples are indicators of a low adaptability level.

\section{The increase of task's complexity level}

\begin{tabular}{|c|c|c|c|}
\hline \multicolumn{2}{|c|}{ Division } & \multicolumn{2}{|c|}{ Addition } \\
\hline Correct & Incorrect & & Incorrect \\
\hline $\begin{array}{l}\text { Quick solution } \\
\text { Short intermediate } \\
\text { operations } \\
\text { Theta-band } \\
\text { maintenance and alpha- } \\
\text { band desynchronization }\end{array}$ & $\begin{array}{l}7 \text { Long-term solution } \\
\text { Long intermediate } \\
\text { operations } \\
\text { Significant } \\
\text { desynchronization of } \\
\text { all bands }\end{array}$ & $\begin{array}{l}\text { Quick solution } \\
\text { Short intermediate } \\
\text { operations } \\
\text { Theta-band maintenance } \\
\text { and alpha-band } \\
\text { desynchronization }\end{array}$ & $\begin{array}{l}\text { Long-term solution } \\
\text { Long intermediate } \\
\text { operations } \\
\text { Significant } \\
\text { desynchronization of all } \\
\text { bands }\end{array}$ \\
\hline $\begin{array}{l}\text { Working memory + } \\
\text { long-term memory }\end{array}$ & $\begin{array}{c}\text { Long-term memory }+ \\
\text { the overload of working } \\
\text { memory }\end{array}$ & $\begin{array}{l}\text { Working memory + } \\
\text { long-term memory }\end{array}$ & $\begin{array}{c}\text { Long-term memory }+ \\
\text { the overload of working } \\
\text { memory }\end{array}$ \\
\hline
\end{tabular}

Fig. 7. Diagram of psychophysiological correlates for solving examples of fractions addition and division

\section{Conclusion}

The analysis of behavioral and electrophysiological correlates allowed us to determine the different nature of the solution algorithm for calculating examples of standard fractions addition and division. The PES absence while the answer is incorrect has drawn us to the conclusion about the solution algorithm rigidity and lack of its correction. The key factors for assessing the adaptability level are the solution time, the number, and the duration of stages. Downward deviations in the data values are associated with the dominance of longterm memory (reflects a low adaptability level due to a little adjustment of the algorithm), and to a large extent - with the dominance of working memory and voluntary attention (reflects an increase in adaptability and adjustment of the solution algorithm). The competency of using this particular set of behavioral parameters was confirmed in examining the results of EEG investigations. 


\section{References}

1. K. Mandrick, V. Peysakhovich, F. Rémy, E. Lepron, M. Causse, Biol Psychol. 121(PtA), 62-73 (2016) doi: 10.1016/j.biopsycho.2016.10.002.

2. J., McMullen, B. Brezovszky, G. Rodríguez-Aflecht, N. Pongsakdi, M.M. HannulaSormunen, E. Lehtinen, Learning and Individual Differences 4, 172-181 (2016) doi:10.1016/j.lindif.2016.02.007

3. C.E. Nanu, J. McMullen, P. Munck, M.M. Hannula-Sormunen, J Exp Child Psychol 169, 42-58 (2018) doi: 10.1016/j.jecp.2017.12.011.

4. D. Braithwaite, E.R. Leibb, R.S. Siegler, J. McMullene, Cognitive Psychology 112, 8198 (2019) doi: 10.1016/j.cogpsych.2019.04.002

5. J.L. Booth, K.J. Newton, Contemporary Educational Psychology 37, 247-253 (2012) doi:10.1016/j.cedpsych.2012.07.001

6. R.S. Siegler, G.J. Duncan, P.E. Davis-Kean, K. Duckworth, A. Claessens, M. Engel, M.I. Susperreguy, M. Chen, Psychol. Sci. 23, 691-697 (2012) doi: $10.1177 / 0956797612440101$

7. J. Rodrigues, N.C. Jordan, N. Hansen, Journal of Learning Disabilities 52(6), 480-497 (2019) doi:10.1177/0022219419879684

8. J. Liu, H. Zhang, C. Chen, H. Chen, J. Cui, X. Zhou, NeuroImage 147, 432-446 (2017) doi: 10.1016/j.neuroimage.2016.12.035

9. J.C. Chow, M. Jacobs, Learning and Individual Differences 47, 252-257 (2016) doi:10.1016/j.lindif.2015.12.017

10. E.K. Aydarkin, A.S.: Fomina, J Integr Neurosci 12(1), 73-89 (2013) doi: 10.1142 / S0219635213500088

11. J.R. Anderson, J.M. Fincham, Cogn Sci. 38(2), 322-352 (2014) doi: 10.1111/cogs. 12068

12. M. Verner, M.J. Herrmann, S.J. Troche, C.M. Roebers, T.H. Rammsayer, Front Hum Neurosci 7, 217 (2013) doi: 10.3389/fnhum.2013.00217

13. E. H. Smith, G. Horga, M.J. Yates, C.B. Mikell, G.P. Banks, Y.J. Pathak, C. A. Schevon, G.M. McKhann, B.Y. Hayden, M.M. Botvinick, S.A. Sheth, Nat Neurosci 22(11), $1883-$ 1891 (2019) doi: 10.1038/s41593-019-0494-0.

14. L. Van der Borght, C. Desmet, W. Notebaert, Front. Psychol. 6, 2051 (2016) doi: 10.3389/fpsyg.2015.02051

15. S. Basharpoor, F. Heidari, P. Molavi, Appl Neuropsychol Adult 1-8 (2019) doi: 10.1080/23279095.2019.1632860

16. C. Desmet, I. Imbo, J. De Brauwer, M. Brass, W. Fias, W. Notebaert, Q J Exp Psychol. 65(6), 1059-67 (2012) doi: 10.1080/17470218.2011.648943

17. L. Cragg, S. Richardson, P.J. Hubber, S. Keeble, C. Gilmore, PLoS One 12(12), e0188693 (2017) doi: 10.1371/journal.pone.0188693

18. J. Riddle, J.M. Scimeca, D. Cellier, S. Dhanani, M. D'Esposito, Curr Biol. 30(9), 17481754 (2020) doi: 10.1016/j.cub.2020.02.065.

19. G. Sammer, C. Blecker, H. Gebhardt, M. Bischoff, R. Stark, K. Morgen, D. Vaitl, Hum Brain Mapp. 28(8), 793-803 (2007) doi: 10.1002/hbm.20309

20. B. Griesmayr, W.R. Gruber, W. Klimesch, P. Sauseng, Neurobiol Learn Mem 93(2), 208-15 (2010) doi: 10.1016/j.nlm.2009.09.013 
21. R. Panda, R.D. Bharath, L. George, S. Kanungo, R.P. Reddy, N. Upadhyay, A. Thamodharan, J. Rajeshwaran, S.L. Rao, A.K. Gupta, Brain Cogn 86, 75-81 (2014) doi: 10.1016 / j.bandc.2014.01.018

22. M. D'Esposito, B.R. Postle, Annu Rev Psychol. 66, 115-42 (2015) doi: 10.1146/annurevpsych-010814-015031

23. J. Kim, J. Chey, S.E. Kim, H. Kim, Neurosci Res. 94, 50-61 (2015) doi: 10.1016 / j.neures.2014.12.009

24. J.P. Kim, S.W. Seo, H.Y. Shin, B.S. Ye, J.J. Yang, C. Kim, M. Kang, S. Jeon, H.J. Kim, H. Cho, J.H. Kim, J.M. Lee, S.T. Kim, D.L. Na, E. Guallar, Neurology 85(9), 806-12 (2015) doi: 10.1212 / WNL.0000000000001884

25. S.I. Dimitriadis, N.A. Laskaris, V. Tsirka, M. Vourkas, S. Micheloyannis, Neurosci Lett. 483(1), 11-5 (2010) doi: 10.1016/j.neulet.2010.07.034

26. C.L. Lin, M. Jung, Y.C. Wu, H.C. She, T.P. Jung, Int J Neural Syst. 25(2), 650-660 (2015) doi: 10.1142/S0129065715500045

27. W. Klimesch, Trends Cogn Sci. 16(12), 606-17 (2012) doi:10.1016/j.tics.2012.10.007

28. C. Moisello, H.B. Meziane, S. Kelly, B. Perfetti, S. Kvint, N. Voutsinas, D. Blanco, A. Quartarone, G. Tononi, M.F. Ghilardi, PLoS One 8(6), e65882 (2013) doi: 10.1371 / journal.pone.0065882

29. A.G. Collins, J.F. Cavanagh, M.J.J. Frank, Neurosci 34(13), 4677-85 (2014) doi: 10.1523/JNEUROSCI.3900-13.2014.

30. A. Kamzanova, G. Matthews, A. Kustubayeva, Appl Psychophysiol Biofeedback 45(3), 183-194 (2020) doi:10.1007/s10484-020-09461-4

31. S. Xie, Y. Li, J Integr Neurosci 19(1), 111-118 (2020) doi: 10.31083/j.jin.2020.01.1234

32. A.A. González-Garrido, F.R. Gómez-Velázquez, R.A. Salido-Ruiz, A. EspinozaValdez, H. Vélez-Pérez, R. Romo-Vazquez, G.B. Gallardo-Moreno, V.D. Ruiz-Stovel, A. Martínez-Ramos, G. Berumen, Brain Cogn. 124, 57-63 (2018) doi: 10.1016/j.bandc. 2018.04 .006

33. S.P. Muthukrishnan, S. Soni, R. Sharma, Brain Topogr 33(1), $75-85$ (2020) doi: 10.1007/s10548-019-00739-3 